Meta

Journal des traducteurs

Translators' Journal

\title{
Formation des traducteurs et traduction philosophique
}

\section{Jean-René Ladmiral}

Volume 50, numéro 1, mars 2005

Enseignement de la traduction dans le monde

Teaching Translation Throughout the World

URI : https://id.erudit.org/iderudit/010660ar

DOI : https://doi.org/10.7202/010660ar

Aller au sommaire du numéro

Éditeur(s)

Les Presses de l'Université de Montréal

ISSN

0026-0452 (imprimé)

1492-1421 (numérique)

Découvrir la revue

Citer cet article

Ladmiral, J.-R. (2005). Formation des traducteurs et traduction philosophique. Meta, 50(1), 96-106. https://doi.org/10.7202/010660ar

\section{Résumé de l'article}

Il semble difficile de structurer la formation des traducteurs dans la perspective d'une pédagogie par objectifs, dans la mesure où cela supposerait que l'activité traduisante peut être analysée comme étant la mise en oeuvre d'un faisceau de compétences partielles ; alors qu'on est sans doute fondé à penser que la compétence traductive correspond à une pratique globale, holiste, et qu'il conviendra donc de mettre en place une formation fondamentale polyvalente et générative. Il reste qu'il y a lieu de faire l'inventaire des divers éléments de formation qui concourent à définir le cursus au terme duquel on aura formé un traducteur professionnel. Dans ce cadre, il se trouve que, très paradoxalement, la traduction philosophique peut apporter une contribution spécifique à la formation des traducteurs professionnels.
Ce document est protégé par la loi sur le droit d'auteur. L’utilisation des services d’Érudit (y compris la reproduction) est assujettie à sa politique d'utilisation que vous pouvez consulter en ligne.

https://apropos.erudit.org/fr/usagers/politique-dutilisation/ 


\title{
Formation des traducteurs et traduction philosophique
}

\author{
JEAN-RENÉ LADMIRAL \\ Université de Paris-X-Nanterre, Paris, France \\ ISIT, Paris, France
}

\begin{abstract}
RÉSUMÉ
Il semble difficile de structurer la formation des traducteurs dans la perspective d'une pédagogie par objectifs, dans la mesure où cela supposerait que l'activité traduisante peut être analysée comme étant la mise en œuvre d'un faisceau de compétences partielles; alors qu'on est sans doute fondé à penser que la compétence traductive correspond à une pratique globale, holiste, et qu'il conviendra donc de mettre en place une formation fondamentale polyvalente et générative. II reste qu'il y a lieu de faire l'inventaire des divers éléments de formation qui concourent à définir le cursus au terme duquel on aura formé un traducteur professionnel. Dans ce cadre, il se trouve que, très paradoxalement, la traduction philosophique peut apporter une contribution spécifique à la formation des traducteurs professionnels.
\end{abstract}

\begin{abstract}
It seems rather difficult to organize the training of translators in the perspective of purpose(s)-oriented didactics (pédagogie par objectifs), for it would imply that translation activity can be analysed as if it were a synergy of different partial competences. It might be assumed, though, that translation competence is a global, holistic, practice; and that we should therefore assert a polyvalent and generative "formation fondamentale." Anyway, it will be necessary to list the different training modules that help define the curriculum aimed at training professionnal translators. Paradoxically, it is to be assumed that philosophical translation can contribute specific ally to their training.
\end{abstract}

\section{MOTS-CLÉS/KEYWORDS}

compétence traductive, didactique, formation (formation fondamentale et formation professionnelle), traduction philosophique

à Claudette Oriol-Boyer

1. Traditionnellement, on oppose traduction littéraire et traduction technique; et il semble en effet difficile de ne pas en revenir finalement toujours à ce clivage binaire. Il reste que plusieurs tentatives ont été faites pour mettre au point une typologie de la traduction plus élaborée: en intégrant la traduction des textes publicitaires et « opératifs» comme troisième type de traduction (Reiss 1976, 2002, etc.), en partant de la pluralité des fonctions communicationnelles du texte à traduire (Matt, Thiel, Thome, Wilss 1978), en thématisant la traduction philosophique comme type spécifique de traduction (Ladmiral 1983a), etc.

Or, l'intérêt de la traduction philosophique n'est pas seulement descriptif: elle constitue un cas limite de la traduction de ce qu'on peut appeler de façon générique le discours théorique. Il lui revient donc un domaine d'application extrêmement large, beaucoup plus que la seule philosophie (voir traduction "générale», traduction 
scientifique, sciences humaines, etc.). Bien plus, le problème posé est celui d'un fonctionnement textuel spécifique, qu'on pourra désigner en ayant recours au concept de métalangage. D’où l'intérêt proprement typologique de ladite traduction "philosophique» (lato sensu).

Par ailleurs, et surtout, il convient de souligner la valeur de la traduction philosophique comme élément de formation fondamentale. Il y a là matière à une didactique à la fois "générative» et polyvalente, générale et professionnalisée. Le dispositif de formation que je me suis attaché à mettre en place se situe entre l'atelier du traducteur et le séminaire de recherche: il a notamment à prendre en compte des paramètres comme l'équation personnelle, l'option traductologique, l'implication institutionnelle, etc.; il entreprend d'aller au-delà de la seule linguistique appliquée et les théorèmes pour la traduction qu'il permet de dégager viennent fournir ce qu'on pourrait appeler la «boîte à outils» du traducteur (voir Ladmiral 2002).

On notera que la traduction philosophique fonctionne aussi comme tertium quid permettant de mieux prendre la mesure de la double dimension à la fois «littéraire» et «technique» inhérente à tout texte. On notera aussi que, de bien des façons, le discours des sciences humaines est omniprésent dans les textes modernes, et qu'en dernière instance, c'est encore de conceptualisation philosophique qu'il s'agit alors: la traduction ayant à y arbitrer l'interférence entre composantes linguistique et lexico-sémantique, mais aussi culturelle et historique, socio-culturelle, voire " ethnoculturelle», et proprement conceptuelle, c'est-à-dire rationnelle. Surtout, il n'est pas de texte à traduire qui ne comporte des éléments de ce «métalangage » qu'il revient à la traduction philosophique de prendre pour objet.

2. Voilà, donc, quelques-uns des principes traductologiques qui me conduisent à recommander l'utilisation de la traduction philosophique dans la perspective d'une didactique de la formation des traducteurs. Au reste, c'est dans le cadre d'une réflexion d'ensemble sur la traduction philosophique que s'inscrivent aussi les pages qu'on va lire, dans la foulée de plusieurs études parallèles portant respectivement sur la typologie de la traduction, sur les éléments d'une traductologie du discours philosophique, sur l'herméneutique philosophique qu'appelle la traduction des philosophes et, plus généralement, sur la dimension philosophique de la traduction elle-même, etc. Autant d'approches, dont j'ai indiqué ailleurs comment elles s'articulent les unes aux autres.

Il y a là, on l'aura pressenti, tout un domaine de recherche et de réflexion dont on pourra appréhender l'ampleur à travers la formulation de ce qu'en rhétorique il est convenu d'appeler une antimétabole, à savoir: de la traduction de la philosophie à la philosophie de la traduction... Mais il est clair que ce n'est pas le lieu d'en traiter ici de façon thématique, car ce n'est qu'incidemment notre objet. C'est pourquoi je me contenterai de renvoyer aux différentes études que j'ai publiées sur cette problématique, en n'en citant ici que deux, où l'on pourra trouver les éléments d'un bilan synthétique de cette recherche qui m'occupe parallèlement depuis quelque trois décennies et, bien sûr, des références bibliographiques plus détaillées (Ladmiral 1998/2004).

À première vue, un tel programme de recherche sur la traduction philosophique pourra faire figure de «spécialité» excentrique (Spezialität), un peu comme un plat exotique et inconnu que ne se risqueront à prendre au menu que quelques rares convives téméraires! En l'occurrence, ce serait l'équivalent intellectuel d'une telle 
incartade culinaire; au point qu'on se trouverait fondé de reprocher à un tel projet de n'obéir qu'à la logique académique visant à définir un domaine de recherche sui generis en fonction des seules finalités propres à l'institution universitaire, voire des seuls intérêts personnels de tel ou tel enseignant-chercheur (en l'occurrence moimême!) au terme d'un plaidoyer pro domo - et ce, sans prendre en considération les échéances de la pratique, les exigences de la formation professionnelle de nos étudiants et la logique de la "demande sociale».

En fait, paradoxalement, il y a une pertinence traductologique de la traduction philosophique qui va bien au-delà du strict secteur du discours philosophique proprement dit, comme je viens de l'indiquer précédemment, d'entrée de jeu, pour ce qui est du fonctionnement linguistique des textes et des extrapolations à la traduction en général qu'il y a donc lieu de dégager à partir de la traduction philosophique. Cela dit, je me situe ici «en aval» des considérations exposées plus haut: j'entends souligner l'aspect pratique de la traduction philosophique, au niveau d'une approche didactique, tant il est vrai que parler de la traduction philosophique, ce n'est pas «sortir des limites de l'épure». Au demeurant, c'est pour moi une conviction déjà ancienne: elle faisait déjà l'objet de ma communication à un colloque de l'A.I.L.A. organisé par Wolfram Wilss à Sarrebruck, à laquelle la présente étude fait très directement écho (voir Ladmiral 1983b) et qu'elle prolonge. En l'occurrence, j'entends bien séparer le registre du «discours didactique» et le registre du «discours scientifique» (voir Ladmiral 1971: 168 sqq.): le premier traitant ici de la formation des traducteurs; alors que le second est celui de la recherche, lequel s'en tient trop souvent, dans le cadre de bien des colloques sur la traduction, à traiter de façon approfondie des points de détail, qui relèvent de ce que je me suis permis de critiquer sous le nom de «micrologie» traductologique.

Mais, s'agissant d'un sujet quand même encore relativement nouveau (et compte tenu des limites imparties à la présente étude), je suis condamné à en rester ici au niveau des principes, me réservant de présenter ultérieurement de façon détaillée le dispositif pédagogique concret qui permet d'assurer la mise en ouvre des principes didactiques évoqués ici. De plus, la perspective adoptée étant justement celle d'une didactique de la traduction, les propositions sur lesquelles on débouche ne peuvent pas être autre chose que des options pédagogiques et ces dernières comportent nécessairement un irréductible noyau « décisionnel », quand bien même on pourra (et on devra) invoquer certains éléments de plausibilité psychologique à l'appui des orientations proposées. Aussi ne devra-t-on pas s'étonner de ce que le discours que je tiens ici comporte les éléments de ce qui apparaîtra, à juste titre, comme un plaidoyer pour la traduction philosophique.

3. S'agissant de didactique en général et, en l'espèce, de la formation des traducteurs en particulier, on pourra adopter grosso modo deux attitudes. D'aucuns tendront à formuler, voire à formaliser d'abord une théorie générale de la traduction, puis à en dégager ensuite les conséquences au niveau de l'application didactique. Pour ma part, je me résous à adopter le point de vue moins ambitieux de l'empirisme, pour des raisons épistémologiques qui tiennent au statut du discours traductologique et à ses limites (Ladmiral 2002: 211 sqq. et passim).

C'est simplement un modèle didactique global de curriculum pour la formation des traducteurs que je mets en avant d'emblée, pragmatiquement; et c'est de son inser- 
tion expresse au sein de cet ensemble que l'enseignement de la traduction philosophique pour lequel je plaide tire sa justification. D’une façon générale, je pars du principe que, pour former des traducteurs, il y a lieu de mobiliser trois types d'éléments de formation et sans doute ce trivium didactique de la traduction fera-t-il assez aisément consensus (quitte à ce que chacun fasse varier la part revenant respectivement à chaque enseignement dispensé et y apporte, éventuellement, quelques éléments de complément).

Dans ce type de "règle des trois tiers», comme celle qui est évoquée ici, on a affaire en général à des tiers «à la Pagnol », c'est-à-dire des «tiers » inégaux... - Ainsi le premier tiers envisagé fait-il, à lui seul, près de $50 \%$ de la formation d'ensemble. Il concerne les exercices de traduction eux-mêmes, avec une part importante de traduction de spécialité, c'est-à-dire de «traduction technique» (lato sensu) ou, pour reprendre une formule de Daniel Gouadec de «traduction à orientation spécialisée»; et je précise bien d'emblée que si je propose ici d'exercer la traduction sur le discours philosophique, ce n'est pas en tant que ce dernier peut être regardé comme une langue de spécialité: méthodologiquement parlant, ce sont là deux choses bien différentes, puisque c'est à la formation fondamentale qu'est censée servir la traduction philosophique dans le contexte qui nous occupe (voir inf.). En outre, au rebours de l' «air du temps», qui pousse à la professionnalisation, et de l'idéologie techniciste qui nous assiège, il conviendra de ne pas négliger les enseignements de traduction générale: paradoxalement, c'est devenu encore plus nécessaire de nos jours, dès lors que la maîtrise de la langue maternelle est de plus en plus incertaine dans les jeunes générations et, à travers elle, la maîtrise de la fonction du langage lui-même.

Bien sûr, ce premier «tiers» (traduction) est lui-même subdivisé en fonction des langues utilisées. Il s'agit là d'un entraînement (training) plus ou moins intensif dont la nécessité et l'importance sont bien évidentes et dont les modalités sont à préciser en fonction de critères pour ainsi dire «techniques», qui ressortissent à une programmation pédagogique et linguistique des contenus d'enseignement, au contrôle «docimo-pédagogique» (feed-back) des procédures et des objectifs, etc. Sans doute certains seront-ils portés à voir là un aspect de la formation des traducteurs justiciable d'une méthodologie didactique proche de l'enseignement programmé et de la psychopédagogie béhaviouriste qui lui est associée. On verra qu'à mon sens, ce ne peut être le dernier mot d'une didactique de la traduction et qu'il convient de faire une place à la dimension complémentaire d'une «formation fondamentale » : d'où, notamment, la place que j'accorde à la traduction philosophique.

4. Le deuxième «tiers» concerne ce que j'appellerais les disciplines auxiliaires (Hilfswissenschaften) de la traduction que devra comporter la formation des traducteurs.

$\left.1^{\circ}\right)$ D'abord et surtout: parmi ces «sciences auxiliaires» de la traduction, la première place revient à la terminologie; et, de fait, on peut dire que nous formons des «traducteurs-terminologues». Point n'est besoin d'insister, car il y a là une évidence acquise qui fait consensus (et il n'est que de consulter les différents articles et numéros de Meta consacrés plus ou moins directement aux différents aspects de cette problématique).

$2^{\circ}$ ) Il est clair aussi qu'on ne saurait traduire sans mobiliser des informations «civilisationnelles». Il s'en est suivi que d'aucuns ont voulu voir dans la traduction une modalité de la communication interculturelle, voire «transculturelle». Sans 
méconnaître cette dimension du problème, à laquelle j'ai consacré tout un travail de recherche-action «sur le terrain» (Ladmiral/Lipiansky 1995: 21-76, Ladmiral 1982, etc.), je pense qu'il faut en limiter l'importance dans le contexte qui nous occupe: il n'y a pas là quelque chose de radicalement nouveau, qui serait venu changer la nature de la traduction. Sans doute convient-il en outre de distinguer ici deux aspects différents. D’une part, il y a lesdites informations civilisationnelles indissociables du référent lui-même, de ce dont traite le texte à traduire, qui permettront de décoder la dimension socio-culturelle du texte-source et de déchiffrer le non-dit de son contexte implicite, quitte à devoir l'expliciter en langue-cible. D'autre part, il est clair qu'on ne traduit pas seulement d'une langue-source (Lo) à une langue-cible (Lt), mais bien d'une langue-culture (LCo) à une autre langue-culture (LCt) : il y a en effet une dimension culturelle attachée substantiellement à toute langue et, plus précisément, à tout état de langue; c'est là ce qu'en 1972 j'avais thématisé en termes de périlangue (voir Ladmiral 2002: 61 et passim). Mais je dirai surtout que ce deuxième aspect (ainsi que le premier, dans une certaine mesure) relève du «bagage cognitif» (Seleskovitch et Lederer 1984) qui fait nécessairement partie du «cahier des charges» inhérent à tout apprentissage sérieux et approfondi d'une langue étrangère, ce qui n'est donc pas fait pour surprendre un traducteur digne de ce nom!

$3^{\circ}$ ) Il n'est que trop évident que le futur traducteur doit aussi maintenant avoir bénéficié d'une formation à l'utilisation des outils informatiques, allant de l'interrogation des bases de données au maniement de divers logiciels, sans parler d'Internet. C'est un défi que s'attachent à relever nos écoles de traduction, à l'instar d'un Daniel Gouadec qui, en cette affaire, a eu à Rennes un rôle de pionnier (voir Gouadec 1994) avant de venir faire profiter l'ISIT de ses lumières. Cela dit, on notera que les dites nouvelles technologies de l'information et de la communication (NTIC) font converger, d'une part, la séquence informatique - qui va d'une sensibilisation à la traduction automatique (TA), c'est-à-dire en fait plus précisément à ce qu'il convient mieux d'appeler la traduction assistée par ordinateur (TAO), à l'utilisation du traitement de textes (TDT) et à l'utilisation des banques de données, tout cela tendant à la redéfinition de la station de travail du traducteur (voir Clas et Safar 1992), sans parler de tout ce qu'on met sous l'appellation de «localisation» - et, d'autre part, le complexe du «multimédias» : ces deux univers technologiques tendant à ne plus faire qu'un dans la pratique. Yves Gambier a insisté sur le fait que cela ne va pas sans conséquences quant au concept même de traduction qui, du coup, s'en trouve considérablement «élargi » et redéfini, ainsi que par là même en ce qui concerne les divers «métiers de la traduction» auxquels il nous revient de préparer nos étudiants (Gambier 2000 et Ladmiral 1995).

$4^{\circ}$ ) Par ailleurs, la question se pose de savoir dans quelle mesure il n'y a pas lieu d'ajouter à tout cela des compléments de formation dans le domaine de la technologie - ou plutôt: des technologies, au pluriel; et c'est cela qui fait problème. Mais il n'entre pas dans mon propos d'en traiter ici. Une remarque seulement: on ne peut ignorer qu'il y a là une masse de savoir telle que, tendanciellement, c'est indominable. Il faudra donc se résoudre à prendre des options partielles. Pour permettre un accès (au moins partiel, donc) à ce savoir référentiel ou «sigmatique», touchant les 
domaines objectifs dont il est question (subject matter), il m'apparaît qu'il y a essentiellement deux (ou trois) stratégies possibles.

La première possibilité irait du côté de la spécialisation et constituerait ce qu'on pourrait appeler l'option «lourde». Ainsi un Reiner Arntz a-t-il en son temps mis en place des enseignements de mécanique (Maschinenbau) dans l'Institut de formation des traducteurs dont il avait pris la direction au sein de la jeune Université de Hildesheim, par exemple. Aussi conviendra-t-il d'envisager une harmonisation de ces choix de formation - sur le plan institutionnel et à une échelle non pas seulement nationale mais internationale (compte tenu des moyens nécessairement limités dont on dispose), voire seulement européenne dans un premier temps - pour assurer une complémentarité entre les différentes Écoles de traducteurs et tendre à "couvrir ", au bout du compte, la majeure partie de ces domaines technologiques. Il est clair qu'il y a là une échéance nécessaire. Toutefois, il semble aussi que, compte tenu de l'ampleur des informations à maîtriser, il faille ne voir dans ces éléments de formation que des réalisations types (exemplarisch), présentant un intérêt méthodologique (en sorte qu'il y aurait paradoxalement une analogie avec le cas limite de la traduction philosophique mais, comme il a été indiqué, il existe entre ces deux types d'enseignements une différence méthodologique essentielle dans le cadre didactique que je propose ici).

Mais il est également une autre possibilité que cet approfondissement unilatéral partiel d'une discipline: on pourra aussi ne viser qu'à une sensibilisation plurielle «en surface», selon une méthode analogue à ce que les pétroliers appellent le carottage. C'est ainsi qu'à l'ISIT, par exemple, il a été mis en place différents ateliers techniques: sur la traduction médicale, le sous-titrage, la localisation, la terminologie informatique, etc. Ces ateliers n'étant pas des options au choix, mais constituant un éventail d'initiations auxquelles participent l'ensemble des étudiants de la promotion. La finalité en est de donner aux futurs traducteurs en formation une idée, précise et concrète, de la diversité des métiers de la traduction auxquels ils seront confrontés. Fondamentalement, ces ateliers ont pour objectif de les entraîner à anticiper des échéances professionnelles très diverses dont, par ailleurs, il est bien clair qu'il ne peut être question de les balayer toutes, si nombreux et diversifiés que puissent être lesdits ateliers (dont la liste ci-dessus n'est qu'indicative). Plutôt que d'acquérir l'intelligence approfondie d'un domaine spécifique (voir sup.), il s'agira de promouvoir une aptitude à innover et à s'adapter à des conjonctures inconnues - qui, du coup, le seront moins, au moins par récurrence analogique. Cela rentre dans le cadre de la didactique à la fois «générative» et polyvalente pour laquelle je plaide dans la présente étude (voir sup.). Plus spécifiquement: il y a sans doute un niveau de semispécialisation qui correspond au besoin propre du traducteur et qui, le cas échéant, lui permettra de consulter efficacement les bases de données disponibles ou de poser des questions ciblées à une personne-ressource (voir Moskowitz 1972). Cette option d'un apprentissage "léger» correspond à ce que, dans un autre contexte, j'avais thématisé comme étant l'équivalent méthodologique de l'équipement alpin auquel certains préfèrent avoir recours dans leurs ascensions himalayennes... (Ladmiral 1988: 153).

À peine est-il besoin de mentionner une troisième possibilité car nos écoles y ont recours quasiment depuis toujours, à savoir : les stages en entreprise (qui devront donner lieu à un rapport de stage). Leur fonction est de familiariser nos étudiants 
avec la réalité de l'entreprise, avec l'environnement institutionnel des métiers de la traduction; et bien sûr, c'est aussi du même coup une façon de faire "entrer» le stagiaire dans un domaine des realia auxquels réfèrent les textes qu'il aura à traduire.

5. C'est bien sûr le troisième «tiers» qui nous intéresse ici: c'est un «petit tiers», en quantité d'heures d'enseignement, mais il m'apparaît essentiel à la formation de nos traducteurs, d'un point de vue qualitatif. Il correspond à ce que j'appelle la «formation fondamentale» et s'articule selon les deux pôles de la théorie et de la pratique.

$\left.1^{\circ}\right)$ D'abord, en effet, il ne me paraît pas inutile d'enseigner la théorie de la traduction ou "traductologie» (Übersetzungswissenschaft). Cela n'est pas évident et ne va pas sans poser quelques problèmes : il restera à définir les modalités d'un tel enseignement et notamment le dispositif à mettre en place. J'en traiterai ultérieurement dans le cadre d'une prochaine étude, où je thématiserai ce que je pense devoir être le "séminaire de traductologie», à partir de ma propre pratique pédagogique (à l'ISIT, et ailleurs). Au reste, ce devrait être le thème d'un prochain numéro de Meta...

$2^{\circ}$ ) Sans doute convient-il d'appuyer les enseignements de réflexion traductologique sur un minimum de connaissances concernant l'histoire de la traduction, en prenant appui notamment sur les travaux de Georges Mounin, de Jean Delisle, de Michel Ballard, de Lieven d'Hulst, de Christian Balliu et d'autres, dont il y aura lieu de faire la synthèse de façon très didactique et "expéditive»... (Balliu 2002). À tout le moins est-ce l'orientation prise par l'ISIT. Quant à moi, j'y vois d'abord un complément culturel qui, pour les jeunes générations de maintenant, n'est pas du luxe! Surtout, c'est une façon soft d'entrer dans la théorie, à partir d'exemples réels et concrets, et d'espérer ainsi faire pièce aux innombrables formes que peut prendre ce que j'ai appelé l'illusion traductive (Ladmiral 2002).

$3^{\circ}$ ) En revanche, la question se pose de savoir s'il y a lieu d'introduire (ou de maintenir) un enseignement de linguistique, proprement dite, distinct de l'enseignement de la traductologie. Au départ, c'était le choix de l'ISIT et de l'ÉSIT, par exemple; mais la tendance y serait plutôt maintenant à relativiser et à minimiser l'importance de la linguistique dans la formation des traducteurs. Alors qu'OutreRhin, plus d'un cursus de formation s'autorise de l'étiquette de linguistique appliquée (Angewandte Sprachwissenschaft).

$4^{\circ}$ ) Mais, ici, j'entends surtout insister sur le pôle pratique qu'à mon sens il conviendrait de mettre en œuvre, par ailleurs. Il y a là plusieurs possibilités : c'est ce que j'appelle les enseignements «fondamentalistes» de traduction ou enseignements de «traductions fondamentales», parmi lesquels prend place l'enseignement de la dite traduction philosophique dont je me suis fait ici l'avocat.

$5^{\circ}$ ) Enfin, avant d'exposer les principes didactiques dont se soutient la recommandation que je fais d'enseigner la traduction philosophique aux futurs traducteurs, je veux encore mentionner rapidement la possibilité d'envisager, en outre, un type d'enseignements situés à mi-chemin entre le pôle théorique et le pôle pratique dont il vient d'être question, il pourrait s'agir d'enseignements méthodologiques à caractère linguistique, plus ou moins spécialisés, qui mettent en œuvre les acquis de la «grammaire du texte» (Textlinguistik) ou d'autres approches analogues, susceptibles de trouver une application fructueuse dans le domaine de la traduction, selon des procédures à déterminer. Pour ce qui est de ladite Textlinguistik, les recherches menées par nos collègues d'Outre-Rhin avaient pris une indéniable avance il y a quelques 
décennies; aussi ne s'étonnera-t-on pas qu'ils aient entrepris assez tôt d'appliquer ces recherches à la traduction, ainsi qu'un Wolfgang Dressler a pu en esquisser le programme. Un seul exemple, parmi bien d'autres possibles: l'analyse de textes qu'en son temps, à l'Institut de Sarrebruck, Gisela Thiel avait utilisée dans la perspective d'un contrôle docimologique de la progression pédagogique, à partir d'une analyse d'erreurs en traduction (Thiel 1980).

Loin de remplacer les précédents, de tels enseignements viendraient "en plus», selon moi. Par ailleurs, il est permis de se demander s'ils ne seraient pas plus utiles encore aux enseignants eux-mêmes, aux enseignants-chercheurs, qu'aux étudiants qu'ils forment - ce qui, à vrai dire, ne constitue pas une objection rédhibitoire dans la mesure où l'amélioration du «produit» fait, implicitement mais essentiellement, partie de la commande en matière d'enseignement (comme on le voit aussi parallèlement en médecine, où cela pose des problèmes déontologiques autrement plus délicats).

Quoi qu'il en soit de cette dernière aporie, de fait c'est plutôt du point de vue de la psychologie de l'apprenant que j'ai conçu la recommandation pédagogique que je fais ici. Je rejoins en cela ce qui fait maintenant figure de «tarte à la crème » en didactique des langues, à savoir l'opposition (volontiers étiquetée d'un double anglicisme aux connotations modernistes) que l'on campe entre le teaching et le learning, en privilégiant le second par rapport au premier. En tout cas, il conviendra de «ne pas trop charger le bateau»! La tentation de tous les "pédagos» est en effet d'alourdir sans cesse la charge d'enseignements, chacun étant foncièrement convaincu de l'importance capitale d'ajouts constants qui, à terme, risquent de rendre les programmes de travail indominables. Or, nos étudiants ont déjà des emplois du temps très lourds; et il faut leur laisser du temps pour la lecture, pour leurs recherches en documentation et pour la préparation des traductions et autres travaux qu'il leur incombe de nous remettre. À l'opposé, il conviendra tout autant, et même plus, de résister à la tentation de faire une place trop grande à la recherche dans nos écoles, au détriment de la formation professionnelle, qui reste leur finalité première. La recherche en traductologie a sa légitimité: elle contribuera à éclairer, plus ou moins directement, nos enseignements et la pratique elle-même, mais elle doit être bien distinguée du cycle de formation; elle concerne les chercheurs, les enseignants et les étudiants «avancés», une fois le cycle d'études achevé.

6. Pour ces raisons - et notamment parce que j'ai tendance à adopter ce que Carl Rogers appelait l' «attitude centrée sur le sujet », ici centrée sur ces sujets apprenant que sont nos étudiants (learning), plutôt qu'à me laisser impressionner par la masse des savoirs censément à acquérir et donc à transmettre (teaching) dans le cadre forcément limité de l'institution et du temps d'étude imparti - je tiens à mettre l'accent sur la notion de formation fondamentale.

Mais je suis bien conscient que, du même coup, je me retrouve à contre-courant. En effet, je prends le contre-pied de la méta-théorie didactique dominante, dont il apparaît qu'on veuille de plus en plus se réclamer plus ou moins explicitement. Pour un Wolfram Wilss et pour bien d'autres, par exemple, il semble que doivent (et que puissent) être définis avec précision des objectifs psycho-pédagogiques partiels (Lernziele, voire Teilkompetenzen) qui commandent la mise en place des enseignements à dispenser. Fondamentalement, je dirai qu'il y a là deux attitudes de principe qui s'opposent quant à une possible didactique de la traduction; et ce débat, à vrai 
dire, s'inscrit autant et plus dans le champ des dites «sciences de l'éducation» que dans celui de la traductologie.

À cet égard, l'option pédagogique (ou «didactique») à laquelle je me rallie quant à moi est celle d'une didactique d'inspiration "néo-humaniste» (geisteswissenschaftlich-bildungstheoretisch) et "critique», dirais-je en première approximation et en rapprochant deux des trois modèles que distingue et oppose le regretté Herwig Blankertz au sein de la théorie didactique. - Le livre de cet auteur aura été, à mon sens, l'un des ouvrages les plus importants de l'après-guerre en sciences de l'éducation (Blankertz 1969); et je suis heureux de lui marquer ici combien lui est redevable ma réflexion, non seulement touchant une possible didactique de la traduction, mais aussi plus généralement mes travaux en didactique des langues (et ce, même si l'auteur devait ne pas se reconnaître dans les conclusions que j'en ai tirées...).

Je postule en effet qu'en ce qui a trait au problème qui nous occupe ici, et sans préjuger de la discussion épistémologique qui sous-tend cette thèse, les attitudes fondamentales émanant de ces deux modèles ( «humaniste» et «critique») convergent dans leur opposition à l'option positiviste d'une didactique d'inspiration béhaviouriste et techniciste. C'est dans cet esprit que je propose l'alternative d'une «formation fondamentale», que j'ai définie comme formation générale et générative, et dont on pourra dire aussi qu'elle fait écho au principe qu'avait en son temps mis en avant Edgar Faure dans le Rapport de l'UNESCO sur l'éducation dont il a assumé le patronage: «apprendre à apprendre».

Pour aller vite et illustrer mon propos a contrario, c'est par le biais d'une «contreoffensive» répondant à l'objection que d'aucuns ne manqueront pas de faire à une telle prise de position - qu'ils trouveront bien traditionaliste, sinon même « rétro »que j'entends maintenant défendre cette dernière et, avec elle, le corollaire que j'en ai dégagé au niveau d'une didactique de la traduction. Contre la recommandation que je fais d'enseigner la traduction philosophique aux futurs traducteurs, en invoquant une dimension propre à ladite formation fondamentale, on fera valoir précisément que la détermination des fameux «objectifs» de l'enseignement dispensé (Lernziele) resterait par trop vague, voire floue et ambiguë (c'est l'objection explicite qui m'avait été faite par Reiner Arntz, par exemple). À cela, il convient de répondre d'abord très nettement que, s'agissant effectivement d'une option principielle dans le domaine didactique, la notion même de formation fondamentale peut bien être récusée d'emblée. Mais ce ne peut être qu'au nom d'une autre option didactique, laquelle procède elle-même d'un présupposé implicite, au niveau de la méta-théorie psychologique; et ce dernier est d'une généralité telle que sa validation ou sa «falsification » n'est pas (pas encore?) possible en toute rigueur expérimentale. Plus précisément (si l'on peut dire), et si l'on veut rétablir les différents niveaux d'abstraction ou d' "enchâssement» interdisciplinaire: ce serait une méta-méta-théorie que constitue la théorie psychologique sous-jacente à la méta-théorie, ressortissant à la didactique générale, qui est elle-même à l'arrière-plan de la théorie traductologique et didactique (fachdidaktisch) qui, à son tour, oriente nos pratiques respectives d'enseignants et de traducteurs...

Encore une fois, il y a là deux options fondamentales possibles qui s'opposent et il n'est pas d'arbitrage entre elles qui soit strictement scientifique et sans un certain résidu décisionnel. - Concrètement, la définition précise d'objectifs opérationnalisés par l'enseignement implique que soient déterminés auparavant avec exactitude les comportements terminaux (terminal behaviour) partiels que l'on vise à obtenir des 
apprenants. C'est là le présupposé méthodologique, et psychologique, de l'enseignement programmé. D'où le projet analytique de «factoriser» tout l'enseignement de la traduction en fonction de tels objectifs ponctuels et circonstanciés; mais il n'est nullement garanti que ce soit la seule et exclusive didactique adéquate. D’abord, il faudrait au moins qu'on pût disposer déjà de critères d'évaluation fiables concernant la qualité des traductions, qui permissent de mesurer avec précision les fameux «comportements terminaux», ce qui est loin d'être le cas. Surtout, il faudrait admettre que l'activité traduisante soit une tâche analysable en termes de performances partielles, clairement observables et mesurables, qui constitueraient autant de comportements terminaux isolables, et c'est une hypothèse qui reste à établir.

7. À vrai dire, l'hypothèse opposée, dont je me réclame, n'est pas démontrée non plus. Mais il est possible d'invoquer en sa faveur de nombreux éléments de plausibilité psychologique, que le manque de place m’empêche de développer ici. Je me contenterai de poser que la «compétence traductive» (translatorische Kompetenz) est une praxie de nature globale, plutôt qu'un montage d'opérations partielles et séquentialisées dont les mécanismes psycholinguistiques élémentaires pourraient faire l'objet d'une mise à plat analytique, avant qu'une didactique «scientifique » de la traduction entreprît de le remonter sous la forme hiérarchisée d'un algorithme de traduction efficace.

Corollairement, je postule qu'il s'opère un transfert de la compétence acquise au contact de la traduction philosophique, qui trouve ainsi à s'appliquer indirectement aux autres registres plus ou moins «techniques » de la traduction. Là encore, il y aura lieu (et matière) d'invoquer les considérations psychologiques dont se soutient la pédagogie proposée. Enfin, une fois déterminé le cadre général où il se situe et dont il tire son sens (comme nous l'avons fait plus haut), il conviendra de définir positivement ou «de l'intérieur» le dispositif qui permet la mise en œuvre concrète des principes didactiques indiqués ici, à savoir: le Séminaire de traduction philosophique (STPhi). Cela fera l'objet d'une prochaine étude - dont, à vrai dire, j'ai déjà esquissé quelques linéaments in nuce (Ladmiral 1987: 16-20).

\section{RÉFÉRENCES}

Awaiss, H. et J. Hardane (dir.) (1999): Traduction: Approches et Théories, Beyrouth, Université Saint-Joseph, coll. «Sources-Cibles».

Balliu, C. (2002): Les traducteurs transparents. La traduction en France à l'époque classique, Bruxelles, Les Éditions du Hazard, coll. «Traductologie».

Blankertz, H. (1969): Theorien und Modelle der Didaktik, München, Juventa, coll. « Grundlagen der Erziehungswissenschaften 6 ».

Clas, A. et H. SAFAR (dir.) (1992): L'Environnement traductionnel. La station de travail du traducteur de l'an 2001 (Actes du colloque de Mons, 25-27 avril 1991), Montréal, AUPELF/Presses de l'Université du Québec, coll. "Actualité scientifique».

Gambier, Y. (2000): «Le traducteur des multimédias: une nouvelle identité professionnelle», dans Mejri, S., Clas, A., Baccouche, T. et G. Gross (dir.), La traduction: théories et pratiques, Actes du colloque international «Traduction humaine, traduction automatique, interprétation ", Tunis 28-30, septembre 2000, vol. I, p. 111-121.

Gouadec, D. (1994) : «Traduction et informatique: les implications pour la formation», Langages 116, p. 59-74.

Gouadec, D. (2002): Profession: traducteur, Paris, La Maison du Dictionnaire. 
Ladmiral, J.-R. (1971): «Le discours scientifique», Revue d'Ethnopsychologie 2-3, p. 153-191.

Ladmiral, J.-R. (1982): «Traduction et psychosociologie», Meta 27-2, p. 196-206.

Ladmiral, J.-R. (1983a): «La traduction philosophique», Revue de Phonétique Appliquée 66-6768 , p. 233-259.

LadmiRal, J.-R. (1983b) : «Traduction philosophique et formation des traducteurs», Translation Theory and its Implementation in the Teaching of Translating and Interpreting, Tübingen, G. Narr Verlag (Tübinger Beiträge zur Linguistik nº 247), p. 231-240.

Ladmiral, J.-R. (1987): «Pour la traduction dans l'enseignement des langues: "version" moderne des Humanités", Les Langues modernes, 1, p. 9-21.

Ladmiral, J.-R. (1988): «Une interlangue interlinguistique», Problèmes théoriques et méthodologiques de l'analyse contrastive (Actes du colloque 29-30-31 octobre 1986), in E. Pietri (dir.), Paris, Publications de la Sorbonne nouvelle, p. 73-98.

Ladmiral, J.-R. (1995): «Traduire, c'est-à-dire... - Phénoménologie d'un concept pluriel», Meta 40-3, p. 409-420.

Ladmiral, J.-R. (1998) : «Principes philosophiques de la traduction», Encyclopédie philosophique universelle, sous la dir. d'André JАСОВ : t. IV, Le Discours Philosophique, sous la dir. de JeanFrançois Mattéi, Paris, PUF, p. 977-998.

Ladmiral, J.-R. (2002): Traduire: théorèmes pour la traduction, Paris, Gallimard, coll. «Tel», nº 246.

Ladmiral, J.-R. (2004): Filosofía de la traducción, Presses de l'Université de Malaga (sous presse).

Ladmiral, J.-R. et E. M. Lipiansky (1995): La Communication interculturelle, Paris, Armand Colin (Bibliothèque européenne des sciences de l'éducation).

Matt, P., Thiel, G., Thome, G. et W. Wilss (1978) : «Übersetzungsrelevante Typologie deutscher und französischer Texte», Zeitschrift für germanistische Linguistik 6.2, p. 223-233.

Mosкowitz, D. (1972) : «Enseignement de la traduction à l'ESIT», Langages 28, p. 110-117.

Reiss, K. (1976): Texttyp und Übersetzungsmethode. Der operative Text, Kronberg/Taunus, Scriptor (Monographien Literatur + Sprache + Didaktik 11).

ReIss, K. (2002): La critique des traductions, ses possibilités et ses limites, traduit de l'allemand par Catherine Bocquet, Arras, Artois Presses Université, coll. «Traductologie».

Seleskovitch, D. et M. Lederer (1984): Interpréter pour traduire, Paris, Didier Érudition, coll. «Traductologie».

ThieL, G. (1980) : «Übersetzungsbezogene Textanalyse als Kontrollinstrument für den Lernfortschritt im Übersetzungsunterricht», Angewandte Übersetzungswissenschaft. Internationales Kolloquium an der Wirtschaftsuniversität Aarhus/Dänemark, Poulsen, S.-O. und W. Wilss (eds.), Århus (Danemark), p. 18-32.

WiLss, W. (1992): Übersetzungsfertigkeit. Annäherungen an einen komplexen übersetzungspraktischen Begriff, Tübingen, G. Narr (Tübinger Beiträge zur Linguistik 376).

N.B.: Conformément à un usage de plus en plus répandu dans les publications en sciences humaines, et qu'on peut trouver agaçant, j'ai été amené à citer plusieurs de mes propres travaux: la présente étude s'inscrit en effet dans le cadre d'une réflexion d'ensemble, dont c'était l'occasion de faire apparaître la cohérence, et avec laquelle il m'a semblé utile de marquer certains points de contact. C'était ainsi une façon d'alléger cette même étude, compte tenu des limites imparties. Et puis, je suis quant à moi reconnaissant aux auteurs que je lis quand ils me fournissent des indications de cette nature, qui me permettent d'approfondir tel ou tel point. Enfin, ce m'a été souvent l'occasion de mentionner quelques numéros spéciaux de revues et autres publications collectives consacrés aux thèmes abordés qu’autrement, peut-être, le lecteur eût ignorés. En revanche, je me suis limité à très peu de choses pour ce qui est des références bibliographiques en général. 